\title{
ESSAI D'ÉVALUATION DU POTENTIEL DE CRÉATION D'EMPLOIS ASSOCIÉS À LA MODERNISATION DES SYSTĖMES DE GESTION DES DÉCHETS MÉNAGERS
}

\author{
Guillaume David et Jacques Desproges \\ Bureau d'études Terra
}

\begin{abstract}
Cet article présente une méthodologie de comptabilité en emplois des performances territoriales de gestion des déchets ménagers, appliquée à l'incidence en emploi de la mise en place de collectes sélectives de déchets ménagers. La méthode est appliquée à un territoire fictif de 100000 habitants. Cette application se base sur la structuration des emplois par étage technique, compte tenu des effets de substitution d'emplois d'un étage à l'autre en fonction de la distribution des flux de déchets. Sont ensuite présentés les principaux résultats d'une extrapolation nationale, une fois les contextes locaux d'exploitation neutralisés. A travers la prépondérance de la fonction tri comme fonction majeure de créations d'emplois dans les dynamiques de modernisation, se dégage un enseignement clair : c'est l'accroissement du taux de valorisation matière qui « tire » l'emploi de gestion des déchets ménagers.
\end{abstract}

This article presents a methodology of employment accounting, related to territorial household refuse policies. The methodology is applied to a fictitious territory of one hundred thousand people. Its application is based on the distribution of employment by technical stages. Substitution effects of employment from a stage to another one are linked with the distribution of the flow-sheet. After that are presented the main results of a national extrapolation, once local contexts neutralized. Through the prevalence of sorting centers as a employment providing function, we can clearly conclude that the growth of the material valorization rate is the most important means towards benefits employment in waste management.

\section{INTRODUCTION}

La mise en place et la montée en puissance des collectes sélectives (notamment de fractions sèches, en particulier d'emballages) conduit à des besoins supplémentaires de main d'œuvre au niveau des différents étages techniques mobilisés par la gestion séparative des déchets ménagers sur un territoire donné.

Cet article, dans le prolongement de l'étude sur « Les conte- nus en emploi des filières de collecte et de traitement des déchets ménagers et assimilés " réalisée en 1997 par Terra pour l'Ademe ${ }^{[1]}$, se propose d'évaluer le contenu en emplois associé aux activités de la collecte et du tri des emballages ménagers. II montre que le gisement d'emploi le plus important associé aux collectes sélectives n'est pas la collecte proprement dite mais le centre de tri, et dans une moindre mesure le tri à la source (à l'interface entre l'habitant et le service de collecte).

L'analyse se borne au repérage des niveaux et des variations des contenus en emplois directs et indirects d'exploitation, dans leur dimension locale et par extrapolation au niveau national. Les emplois de réalisation des investissements et du recyclage des produits ne sont pas comptabilisés. La question de la solvabilité des emplois et des incidences coûts/emplois de la mise en place des collectes sélectives sera abordée dans une seconde partie.

\section{L'ÉVALUATION MICRO-ÉCONOMIQUE : LES INDICATEURS DE CONTENUS EN EMPLOIS}

\section{Indications de méthode}

Les résultats présentés reposent sur une méthode d'évaluation du contenu en emplois de l'ensemble des technologies de collectes et de traitement des déchets ménagers. Cette méthode est applicable à deux niveaux :

- évaluation des contenus en emplois propres à chaque " étage » ou option technique de gestion des déchets, pris isolément,

- évaluation locale intégrée des contenus en emplois correspondant à la circulation des flux entre tous les étages de collecte et traitement qui caractérisent l'organisation - ou le projet d'organisation - d'un territoire.

La méthode repose sur la segmentation des fonctions structurant une organisation globale de gestion des déchets ménagers en étages techniques, chacun caractérisé par des options techniques ou des effets de contextes particuliers.

Pour chaque étage technique sont précisés des indicateurs de contenus-emplois, les paramètres qui les font spécifi- 
Tableau I : Amplitudes par technique des écarts observés de contenus en emplois

\begin{tabular}{|c|c|}
\hline Techniques & $\begin{array}{l}\text { ICE po } \\
000 \text { tor } \\
\text { par an }\end{array}$ \\
\hline Pré collecte unitaire & 0,06 \\
\hline Pré collecte séparative & 0,07 \\
\hline Communication de proximité & 0,1 \\
\hline Collecte unitaire & 1,4 \\
\hline Collecte sélective PAP & 2,4 \\
\hline Collecte séparative PAP & 1,79 \\
\hline Collecte séparative PAP en simultané & 1,72 \\
\hline Collecte séparative PAP en substitution & 1,54 \\
\hline CS en apport volontaire (verre ou/et JRM) & 0,4 \\
\hline CS en apport volontaire (bi ou tri-flux, avec plastiques) & 0,7 \\
\hline Déchetteries & 0,7 \\
\hline Station de transfert & 0,1 \\
\hline Centre de tri & 3,2 \\
\hline Compostage des déchets verts & 0,3 \\
\hline UIOM & 0,32 \\
\hline MIOM & 0,07 \\
\hline CET 2 & 0,1 \\
\hline CET I & 0,25 \\
\hline \multicolumn{2}{|l|}{$\begin{array}{l}\text { (*)ICE : Indicateur de Contenu emploi, soit l'emploi mesuré en } \\
\text { équivalent temps plein rapporté à } 1000 \text { t/an de flux entrant dans la } \\
\text { fonction technique considérée } \\
(* *) \text { Écarts extrêmes observés pour une même fonction technique } \\
\text { (effets d'options techniques, de contextes socio-géographiques ou } \\
\text { d'exploitation particuliers) }\end{array}$} \\
\hline
\end{tabular}

quement varier et l'amplitude de ces variations. Les contenus en emplois sont rapportés au flux et/ou au dimensionnement des équipements, sur la base d'une unité physique de mesure, soit I 000 tonnes par an.

Les contenus en emplois sont décomposés par fonctions du personnel, en prenant soin d'incorporer un taux de remplacement référentiel du personnel, ainsi que des coefficients d'encadrementmaintenance pour tenir compte des emplois indirects d'exploitation. Les indicateurs de contenus en emplois (ICE) ne constituent pas des indicateurs de productivité du travail mais sont calculés en fonction d'indicateurs moyens de productivité.

Les indicateurs de contenus en emplois sont issus de la confrontation de données empiriques à un modèle analytique. Ils n'ont aucun caractère normatif. Ils constituent des points de repère, correspondant à des organisations rationalisées : il ne s'agit en aucun cas d'optimum théoriques. L'utilité des ICE de référence n'est pas de comparer des situations d'exploitation différenciées, très hétérogènes, mais de dégager des ordres de grandeur utilisables pour des consolidations et comparaisons ultérieures.

\section{Principaux résultats}

Il est ensuite possible d'articuler ces options tech-

\begin{tabular}{|c|}
\hline $\begin{array}{l}\text { Amplitudes des } \\
\text { écarts de } \\
\text { contenu emploi (**) }\end{array}$ \\
\hline 1 à 5 \\
\hline I à 5 \\
\hline 1 à 2 \\
\hline I à 3 \\
\hline I à 3 \\
\hline | à $\mid, 8$ \\
\hline I à $\mid, 2$ \\
\hline 1 à 2 \\
\hline I à 2,8 \\
\hline 1 à 9 \\
\hline I à 16 \\
\hline I à 20 \\
\hline I à 25 \\
\hline | à $\mid, 6$ \\
\hline I à 4,8 \\
\hline I à 4,5 \\
\hline I à 6 \\
\hline I à 1,5 \\
\hline
\end{tabular}

Principaux déterminants de variation

Qualité du service

Qualité du service

Montée en charge ou suivi pérennisé

Milieu et taille

Nombre et composition des flux

Nombre et composition en addition des flux

Nombre et composition des flux

Nombre et composition des flux

Milieu et taille

Nombre et composition des flux

Densité d'implantation et horaires

Taille et fonction (avec ou sans préparation matière)

Nombre et composition des flux

Nature des flux et niveau des apports

Date de mise en service et taille

Taille

Taille

Taille

Tableau 2 : Indicateurs de contenu emploi pour 1000 tonnes par an en fonction d'options ou de contextes particuliers

\begin{tabular}{|c|c|c|c|}
\hline Techniques & $\begin{array}{l}\text { CE pour } \\
000 \text { t/an }\end{array}$ & $\begin{array}{l}\text { Options ou } \\
\text { contextes particuliers }\end{array}$ & $\begin{array}{l}\text { ICE selon } \\
\text { options }\end{array}$ \\
\hline Pré collecte unitaire & 0,06 & $\begin{array}{l}\text { Entretien des bacs } \\
\text { Distribution des sacs }\end{array}$ & $\begin{array}{l}0,054 \\
0,08\end{array}$ \\
\hline Pré collecte séparative & 0,07 & $\begin{array}{l}\text { Entretien des bacs } \\
\text { Distribution des sacs }\end{array}$ & $\begin{array}{l}0,07 \\
0,115\end{array}$ \\
\hline $\begin{array}{l}\text { Communication } \\
\text { de proximité }\end{array}$ & 0,1 & $\begin{array}{l}\text { Montée en charge } \\
\text { Suivi pérennisé }\end{array}$ & $\begin{array}{l}0,2 \\
0,05\end{array}$ \\
\hline Collecte unitaire PAP & 1,4 & $\begin{array}{l}\text { Rural } \\
\text { Semi-urbain } \\
\text { Urbain }\end{array}$ & $\begin{array}{l}1,1 \\
1,3 \\
1,7\end{array}$ \\
\hline Collecte sélective PAP & 2,4 & $\begin{array}{l}\text { Mono-flux avec verre } \\
\text { Mono-flux sans verre } \\
\text { Bi-flux corps creux/corps plats } \\
\text { Bi-flux sans verre }\end{array}$ & $\begin{array}{l}1,9 \\
2,2 \\
2,4 \\
2,8\end{array}$ \\
\hline Collecte séparative PAP & 1,7 & $\begin{array}{l}\text { En substitution } \\
\text { En addition } \\
\text { En simultané }\end{array}$ & $\begin{array}{l}1,54 \\
1,79 \\
1,72\end{array}$ \\
\hline Collecte sélective en AV & $\vee 0,4$ & $\begin{array}{l}\text { Verre en AV } \\
\text { CS AV tri-flux }\end{array}$ & $\begin{array}{l}0,4 \\
0,7\end{array}$ \\
\hline Déchetteries & 0,7 & $\begin{array}{l}\text { I pour } 40000 \text { habitants } \\
\text { I pour } 10000 \text { habitants }\end{array}$ & $\begin{array}{l}0,5 \\
1,2\end{array}$ \\
\hline Station de transfert & 0,1 & $\begin{array}{l}\text { Compactage simple } \\
\text { Préparation matière }\end{array}$ & $\begin{array}{l}0,03 \\
0,15\end{array}$ \\
\hline Centre de tri & 3,2 & $\begin{array}{l}\text { Bi-flux avec verre et JRM en tri négatif } \\
\text { Bi-flux sans verre et JRM en tri négatif } \\
\text { Corps creux hors verre }\end{array}$ & $\begin{array}{l}2 \\
3,4 \\
4,8\end{array}$ \\
\hline $\begin{array}{l}\text { Compostage des } \\
\text { déchets végétaux }\end{array}$ & 0,3 & $\begin{array}{l}\text { Déchets végétaux } \\
\text { Végétaux }+ \text { fermentescibles }\end{array}$ & $\begin{array}{l}0,3 \\
0,4\end{array}$ \\
\hline UIOM & 0,32 & $\begin{array}{l}37500 \mathrm{t} / \mathrm{an} \\
220000 \mathrm{t} / \mathrm{an}\end{array}$ & $\begin{array}{l}0,48 \\
0,19\end{array}$ \\
\hline $\begin{array}{l}\text { Maturation des } \\
\text { mâchefers }\end{array}$ & 0,07 & $\begin{array}{l}50000 \mathrm{t} / \mathrm{an} \\
200000 \mathrm{t} / \mathrm{an}\end{array}$ & $\begin{array}{l}0,15 \\
0,03\end{array}$ \\
\hline CET & 0,1 & $\begin{array}{l}\text { Enfouissement CET } 2 \\
\text { Stabilisation CET I }\end{array}$ & $\begin{array}{l}0,1 \\
0,25\end{array}$ \\
\hline
\end{tabular}


niques sur une logique de filière collecte + traitement. II s'agit de raisonner sur la politique de modernisation dans la « logique 2002 », en augmentant fortement la valorisation matière et énergétique. On mesure les variations de contenus-emplois en fonction d'une complexification croissante des tâches techniques conduisant à des taux de valorisation croissants.

Indépendamment de la méthode produite, cette étude a permis de dégager quelques enseignements de portée générale sur les effets pour l'emploi des dynamiques de modernisation en œuvre dans la gestion des déchets ménagers.

\section{L'ÉVALUATION MACRO-ÉCONOMIQUE : LES EMPLOIS DE LA GESTION DES OM}

\section{Éléments de quantification macro-écono- mique}

La comptabilité en emploi au niveau national repose sur une côte " mal taillée » dans la mesure où les chiffres de I'Insee ne recouvrent que les emplois marchands. Toutefois, une décomposition analytique des emplois par étages techniques de collecte/traitement des OM est possible. Les emplois de collecte et de traitement des OM (hors DIB) se chiffrent à 53000 emplois directs et indirects d'exploitation pour 1997, y

Tableau 3 : Les emplois de la gestion des OM en France pour 1997, en équivalents temps plein

$\begin{array}{lc}\begin{array}{l}\text { Étages techniques } \\ \text { de gestion des OM }\end{array} & \begin{array}{c}\text { Contenu en } \\ \text { emploi (ETP) }\end{array} \\ \text { Collecte } & 40000 \\ \text { Déchetteries } & 3000 \\ \text { Tri } & 5000 \\ \text { Incinération } & 3000 \\ \text { Autres traitements } & 3000 \\ \text { Total } & 53000 \\ \text { Sources : Insee, Ifen, Terra } & (2) \\ \end{array}$
compris les emplois de la récupération, dont $10 \%$ environ sont imputables aux ordures ménagères (le reste des emplois de la récupération concernant les DIB).

\section{OM \\ Répartition des emplois de gestion des}

Dans les filières globales de collecte et de traitement des déchets ménagers, les emplois se séparent schématiquement en deux grands univers et dans les proportions suivantes:

- en amont, les emplois de collectes et de tris représentent $80 \%$ ou davantage des emplois totaux, quel que soit le niveau de complexité de la chaîne globale de collecte et traitement. Ces emplois " amont » de collecte et de tri sont particulièrement réactifs aux variations de volumes et de caractéristiques de flux de déchets ;

- en aval, les emplois de procédé et d'élimination finale - qui représentent 8 à $15 \%$ des emplois totaux. Ils sont quant à eux infiniment moins sensibles que les précédents aux variations de flux.

Les dynamiques de modernisation, bien que multipliant le nombre de technologies de traitement, ne modifient que peu ou pas ce rapport « $85 / 15$ » entre les deux univers d'emplois.

\section{LES EMPLOIS DU TRI DES EMBALLAGES MÉNAGERS}

\section{Paramètres de variation des emplois du tri}

Quel que soit le degré de mécanisation des centres de tri à venir, certaines prévisions énoncées semblent bien optimistes (entre 13000 et 21000 créations d'emplois au niveau national, soit une hypothèse intermédiaire de 17000 créations nettes d'emplois), d'autres peu encourageantes (environ 6000 emplois ${ }^{[3]}$.

Le problème consiste à prévoir la population impliquée dans les collectes sélectives, à différentes échéances, ainsi que les taux de captage atteints par les différents programmes. La montée en charge des programmes incite à la prudence quant aux résultats obtenus.

Le tri fait partie des étages techniques pour lesquels l'amplitude des écarts observés est la plus forte. Toutefois, la généralisation des centres de tri et la multiplication des retours d'expérience laissent supposer une diminution des phases de "tâtonnement " et la résolution de certains points d'incertitudes techniques (schémas de collecte, surdimensionnements, erreurs de conception et d'ergonomie). Une analyse de sensibilité aux paramètres selon différentes configurations de centres de tri indique que les deux principaux paramètres de variation des contenus - emplois du tri sont la nature et la composition des flux d'une part, la productivité du travail d'autre part.

On constate une forte hétérogénéité des contenus en emplois, rapportés aux tonnages traités, en fonction notamment de la présence du verre ou non dans le tonnage trié et les phénomènes de montée en charge des dispositifs. Y compris les emplois de manutention et d'encadrement, 300 à 400 tonnes sont triées par an et par emploi. Cet ordre de grandeur comporte diverses irrégularités ou exceptions notables, surtout si l'on ramène les emplois au tonnage non pas entrant mais valorisé.

En fonction de compositions différenciées de la fraction sélective triée, il est possible d'évaluer le tonnage trié par

\begin{tabular}{|c|c|c|c|}
\hline \multicolumn{4}{|c|}{$\begin{array}{c}\text { Tableau } 4 \text { : Hypothèses référentielles de productivité } \\
\text { du tri manuel, sur la base de I } 700 \text { préhensions } \\
\text { par heure et par trieur }\end{array}$} \\
\hline $\begin{array}{l}\text { Composition } \\
\text { des flux }\end{array}$ & $\begin{array}{l}\mathrm{kg} / \mathrm{h} \\
\text { trieur }\end{array}$ & $\begin{array}{l}\text { Tri } \\
\text { négatif }\end{array}$ & $\begin{array}{l}\text { Taux } \\
\text { de refus }\end{array}$ \\
\hline Mélange avec verre & 160 à 280 & Verre cassé & 20 à $40 \%$ \\
\hline Mélange hors verre & & JRM & 15 à $25 \%$ \\
\hline Bi-flux avec verre & 300 à 600 & Verre cassé/JRM & 20 à $30 \%$ \\
\hline Bi-flux hors verre & 280 à 400 & PET/JRM & 10 à $15 \%$ \\
\hline Corps creux hors verre & 120 à 160 & PET & 10 à $15 \%$ \\
\hline Corps plats & 400 à 700 & JRM & 5 à $10 \%$ \\
\hline Bouteilles plastiques & 60 à 100 & PET & 5 à $10 \%$ \\
\hline
\end{tabular}


trieur et par an. Il convient aussi d'intégrer un taux d'encadrement et manutention proche de $30 \%$. On considère que l'acier est capté par overband.

\section{Les déplacements d'emplois sur l'ensemble de la filière collecte + traitement}

Les effets sur l'emploi sont liés au déplacement des flux et à leur distribution par fonctions (collecte, transfert, tri, élimination du reste des $\mathrm{OM}$ ). Les emplois de tri sont les principaux bénéficiaires des stratégies de valorisation matière. Mais ces stratégies ont aussi des incidences sur les contenus - emplois des autres fonctions de collecte et de traitement.

Sous réserve d'une collecte optimisée des OM résiduelles,

\begin{tabular}{|c|c|c|c|}
\hline \multicolumn{4}{|c|}{$\begin{array}{l}\text { Tableau 5: Emplois de collecte, tri + traitements } \\
\text { (U1OM avec récupération d'énergie) pour } \\
100000 \text { habitants ( } 400 \text { kg/habitant/an) }\end{array}$} \\
\hline & $\begin{array}{l}\text { Collecte } \\
\text { unitaire }\end{array}$ & $\begin{array}{c}\text { CS } 40 \\
\text { kg/hab/an }\end{array}$ & $\begin{array}{c}\text { CS } 80 \\
\text { kg/hab/an }\end{array}$ \\
\hline Emplois collecte OM brutes & 60 & 56 & 52 \\
\hline Emplois collecte sélective PAP & 0 & 8 & 12 \\
\hline Emplois de tri & 0 & 13 & 24 \\
\hline Emplois de traitements & 14 & 13 & 12 \\
\hline Total des emplois & 74 & 90 & 100 \\
\hline Taux de variation des emplois & - & $+20 \%$ & $+35 \%$ \\
\hline Taux de valorisation matière & $0 \%$ & $9 \%$ & $17 \%$ \\
\hline Taux de valorisation globale & $75 \%$ & $84 \%$ & $92 \%$ \\
\hline
\end{tabular}

les effectifs de collecte tendent à se stabiliser une fois les seuils de montée en charge atteints.

En raison d'effets de taille restreints (à productivité constante, l'augmentation du tonnage entrant se traduit par des besoins en main d'œuvre sensiblement équivalents), le contenu en emploi du tri augmente de manière relativement linéaire et proportionnellement aux niveaux d'apport (postes de tri supplémentaires, doublement des équipes, etc.).

Le doublement des rendements de collecte sélective se répercute de manière linéaire sur la part des emplois de tri dans la structure globale des emplois de collecte + traitement, qui passent de $15 \%$ à $25 \%$ du total des emplois. Selon les flux divertis, les emplois de traitement (incinération et gestion des sous-produits) tendent à se réduire d'environ $5 \%$ à $10 \%$.

Le passage d'une configuration unitaire à une configuration séparative de gestion des ordures ménagères se traduit par un accroissement des emplois totaux de l'ordre de $20 \%$ à $35 \%$.

La séparation en 2 ou 3 flux distincts à la source permet d'améliorer les rendements de collecte et la productivité du tri, ce qui ne joue pas en faveur de l'emploi. La généralisation de ce type de schéma de collecte devrait relativiser l'impact des collectes séparatives sur le contenu emploi du tri, qui ne sera pas aussi élevé que les premières estimations le laissent penser.

\section{LES EMPLOIS DU TRI À LA SOURCE.}

\section{Le tri à la source et l'économie domestique}

La séparation en flux distincts à la source implique un certain nombre d'opération préalables, qui se situent en amont même de la collecte : il s'agit d'un ensemble encore récent d'opérations, au plus près de l'habitant, regroupées ici sous le vocable de "tri à la source ».

Les collectes séparatives induisent un temps passé par les habitants (ou les employés de maison) en habitat individuel et par les gardiens, concierges, etc. en habitat vertical. Ces éléments sont difficilement quantifiables en termes de contenus en emplois. De même, l'aménagement des locaux de stockage impliquent des investissements parfois lourds, riches en emplois indirects (travaux d'aménagement des bâtiments, pour mémoire).

Elles induisent aussi la multiplication des récipients présentés à la collecte et génèrent des investissements et des coûts d'entretien qu'il est possible de chiffrer ${ }^{[4]}$. Elles occasionnent des achats de conteneurs d'apports volontaires ou, pour le porte-à-porte, une multiplication des besoins en récipients comprise entre I,7 et I,9 selon les modalités de collecte, le nombre de flux sélectifs, la composition de l'habitat.

Consolidé sur l'ensemble des flux collectés (flux sélectif + reste des OM), l'impact sur l'emploi de la multiplication des contenants est environ de $15 \%$ par rapport à la situation antérieure. La distribution des sacs (là où elle est effectuée), requiert environ un emploi pour 40000 habitants. La mise en place et le suivi d'un parc de contenants de collecte sélective peut mobiliser 2 à 3 personnes pour 100000 habitants, avec un recours massif à l'intérim en période de démarrage.

\section{La communication de proximité associée aux collectes sélectives}

La communication par support écrit étant largement insuffisante à assurer le succès des collectes sélectives, ce poste attire aujourd'hui l'attention, dans un contexte où l'emploi devient une préoccupation collective...

D'après Eco-Emballages, la communication de terrain assure une amélioration des rendements de collecte sélective comprise entre 20 et $45 \%$, sur la base d'un emploi de communication pour 15000 habitants environ ${ }^{[5]}$.

L'enquête réalisée par Terra auprès de 8 collectivités qui ont déjà recours à ce type d'emplois montre que le niveau d'emploi nécessaire est d'environ un emploi pour 8000 habitants en période de montée en charge, contre un pour 30000 habitants en suivi pérennisé, une fois la collecte sélective intégrée par les habitants.

Des obstacles à la pérennisation de ce type d'emploi sont susceptibles d'intervenir... Des retours d'expérience plus nombreux permettraient de comparer des situations de collectes optimisées, avec maintien ou non des équipes de communication. Toutefois, leur maintien une fois les seuils 
de montée en charge dépassés semble techniquement souhaitable.

Une solution consiste à coupler directement la communication au centre de tri. L'équipe se rend dans les endroits où la collecte obtient de mauvais résultats (quantités trop faibles ou mauvaise qualité du tri à la source). Le suivi de la qualité suppose un relevé précis des zones collectées et une attention particulière portée au suivi des flux. Le développement de l'informatique embarquée est susceptible d'améliorer l'articulation collecte/tri.

\section{CONCLUSIONS}

L'impact en emploi des collectes sélectives concerne moins la stricte fonction de collecte (en raison d'optimisations logistiques, une fois les seuils de montée en charge dépassés) que la fonction de tri, étage technique qui n'enregistre pas les économies d'échelle qui caractérisent les techniques d'élimination (incinération et stockage) : un accroissement des performances de valorisation matière se traduit principalement par un accroissement plus ou moins proportionnel des emplois de tri, sans incidences fortes sur le contenu en emplois des autres étages de traitement.

Des objectifs ambitieux de collectes sélectives de fractions sèches supposent la mise en place d'équipes de communication ainsi que l'approfondissement de la réflexion menée par chaque collectivité sur les conditions de leur acceptabilité. Le contenu emploi de la pré-collecte (ou tri à la source) est certes modeste. Sur la base d'un emploi de communication pour 15000 habitants et de 3 emplois de gestion des contenants pour 100000 habitants, le tri à la source peut représenter environ $10 \%$ du total des emplois de collecte + traitement (soit 110 emplois au lieu de 100 dans la simulation sur 100000 habitants proposée plus haut).

Ce type d'emploi dépend fortement des objectifs de valorisation matière énoncés par les collectivités et des moyens confiés à leurs opérateurs. Sur le plan local, le suivi des dispositifs et la mise au point d'outils adaptés, peuvent représenter aussi un volume de travail important.

Sur la base d'une répartition référentielle des effectifs de collecte et de traitement des OM (hors DIB) qui se chiffrent à 53000 emplois environ pour 1997, la potentialité de création d'emploi associée à la réalisation des objectifs 2002 en matière d'emballages des ménages se chiffre à 12000 emplois supplémentaires pour le tri et 6000 emplois supplémentaires pour la collecte, soit 18000 emplois directs d'exploitation additionnels, pour 40 millions de français ( 12 millions étant déjà considérés comme impliqués dans des programmes de collecte sélective des emballages ménagers).

Cette estimation renvoie à un scénario médian au sein duquel le développement des emplois de proximité constitue un levier supplémentaire pour l'emploi. II convient de dissocier emplois de pré-collecte (gestion des contenants) et communication de proximité proprement dite.

Le passage d'une configuration unitaire à une configuration séparative (collecte + tri sélectif) est susceptible d'entraîner, sans autre modification des filières de traitement (dans la mesure où il reste toujours $80 \%$ du tonnage à incinérer ou à enfouir) un accroissement de l'emploi de l'ordre de 20 à $35 \%$, selon les options techniques mises en œuvre. Si l'on y ajoute les postes de pré-collecte et de communication de proximité, l'accroissement global par rapport à une configuration unitaire est de l'ordre de $45 \%$ environ.

Le gisement d'emploi associé à la communication de proximité est de l'ordre de 3500 emplois additionnels (il en existe environ I 000 aujourd'hui). La question se pose du statut de cette main d'œuvre et de sa pérennisation ${ }^{[6]}$. En les intégrant au scénario médian, on obtient un chiffre de 21500 emplois additionnels, imputables à la généralisation des collectes sélectives auprès des 40 millions de français aujourd'hui encore non desservis par la collecte sélective.

\section{Guillaume David et Jacques Desproges}

Bureau d'études Terra - 52, rue de Paradis - 75010 Paris

\section{Bibliographie}

[1] Terra, Le contenu en emplois des filières de collecte et de traitement des déchets ménagers et assimilés, Éditions de l'Ademe, 132 pages, septembre 1998. Ce rapport développe un outil - guide d'évaluation coût/emploi des performances territoriales de gestion des déchets ménagers et assimilés.

[2] Insee, Enquête annuelle d'entreprise, édition 1995 - 90.0B, Enlèvement et traitement des ordures ménagères, p. 87. IFEN, Les données de l'environnement, 1994.

[3] Bertolini G., Déchet, mode d'emploi, Economica, 1996, pp. 96 à 100. Bertolini G., Collecte, traitement, valorisation des ordures ménagères : essai de comptabilité en emplois, Techniques et Sciences Municipales, $n^{\circ} 12$, décembre 1996, pp. 68 à 81 .

[4] Bipe Conseil, Impact des politiques d'environnement sur l'emploi, Mai I996, page 2.

[5] Arene, Les déchets ménagers en habitat vertical. Guide pratique pour la collecte sélective, Cahier technique $n^{\circ} 5,1996$, $84 \mathrm{p}$.

[6] Eco-Emballages, Information et sensibilisation des habitants en matière de collecte sélective : quelques initiatives locales, mars 1996.

[7] Bertolini G., Emplois jeunes : les animateurs de collectes sélectives. Partie I: essai d'analyse économique, Déchets Sciences et Techniques, $n^{\circ} 9, I^{\text {er }}$ trimestre 1998, pp. 33 à 38. 